\title{
Application of point of care transthoracic echocardiography in parturient with peripartum cardiomyopathy undergoing lower segmental cesarean section- 2 cases report
}

\author{
Su MY, Zhang Y, Xia XP, Hu LJ and Li BB* \\ Department of anesthesiology, Drum Tower Hospital, Nanjing University Medical School, Nanjing, China
}

\begin{abstract}
Background: Peripartum cardiomyopathy is one form of life-threatening dilated cardiomyopathy which occurs in previously healthy women in the final months of pregnancy and up to 5 months after delivery. The etiology of peripartum cardiomyopathy remains poorly understood but inflammation or virus- mediated autoimmune reaction to myocardial cells was proposed to be involved in the pathogenesis of peripartum cardiomyopathy. Obstetric management for those parturients requires communication and careful planning between obstetrics, cardiology and anesthesiology.

Case presentation: The first case is a 22 -year-old woman presented as gravida 1 , para 0 , at $38+4$ weeks of gestation with the deterioration of dyspnea on exertion and palpitation over the past several days. According to TTE report from cardiac based echocardiography unit, there was a decline in systolic function manifested by overall global wall motion abnormality with EF only $28 \%$. In the second case, A 44-year-old pregnant woman presented at the same hospital with progressing shortness of breath, chest pain and faintness which was diagnose peripartum cardiomyopathy also.

Conclusion: The application of point of care echocardiography might have a beneficial impact on the selection of anesthesia and maintaining the hemodynamic stability of the parturient with peripartum cardiomyopathy undergoing cesarean section. This paper reported two parturients suffering from peripartum cardiomyopathy with acute heart failure undergoing lower segmental cesarean section uneventfully throughout the perioperative period under the guidance of point of care transthoracic echocardiography.
\end{abstract}

\section{Highlights}

- Intraoperative transthoracic echocardiography (TTE) plays an important role in identifying the undifferentiated causes of dyspnea of parturient with peripartum cardiomyopathy (PPCM), decision making on the anesthetic management and also evaluating the response to therapeutic intervention during the low segmental cesarean section (LSCS).

- Point of care TTE was a useful tool to detect the global or segmental wall motion abnormality of left ventricle as well as right ventricle filling through utility of continuous noninvasive superficial echocardiography in parturients with PPCM undergoing LSCS.

\begin{abstract}
Abbreviations: PPCM: Peripartum cardiomyopathy, EPSS: E point of anterior leaflet of mitral valve septum separation, IVC: inferior vena cava, VTI: velocity time interval, LVOT: left ventricle outflow tract, TTE: Intraoperative transthoracic echocardiography, HFA: The Working Group on PPCM of the Heart Failure Association, ESC: the European Society of Cardiology.
\end{abstract}

\section{Background}

The Working Group on PPCM of the Heart Failure Association (HFA) of the European Society of Cardiology (ESC) recently proposed a new simplified definition of PPCM as an idiopathic cardiomyopathy frequently presenting with heart failure secondary to LV systolic dysfunction (LVEF $<45 \%$ ) towards the end of pregnancy or in the months following delivery, if no other cause of heart failure is found [1]. The reported prevalence of PPCM varied among distinct countries from as low as 1:15,000 live birth in US to as high as 1:400 in Haiti. Patients with PPCM are likely to experience rapid hemodynamic deterioration prominently exhibiting with acute heart failure, malignant arrhythmias, and even death during labor [2-5]. How to conduct a safe anesthesia and guarantee satisfactory recovery for those parturients during low segmental cesarean section (LSCS) is still a big challenge for the anesthesiologist. As it known, the presenting symptoms and signs of PPCM are typically nonspecific and can't be easily distinguished from pregnancy related hypertension, preeclampsia during the peripartum period without an echocardiographic report in operation room. Moreover, the choice of anesthesia (general or neuraxial anesthesia), fluid management strategy, maintenance of cardiovascular stability, or fetus protection for those parturients varies among diverse institutions, no consensus has been reached currently owing to lack of high-quality evidence from clinical trials [6,7].

Intraoperative transthoracic echocardiography (TTE) can provide the real-time assessment of ventricular function, valvular morphology

${ }^{\star}$ Correspondence to: Li BB, Department of anesthesiology, Drum Tower Hospital, Nanjing University Medical School, Nanjing, China, E-mail: icecolor@163.com

Key words: peripartum cardiomyopathy, point of care echocardiography, cesarean section

Received: July 13, 2018; Accepted: July 23, 2018; Published: July 27, 2018 

section- 2 cases report

or volume status of patient, which has been gradually applied in diagnosis or guiding clinical management for patients with circulatory collapse in non-cardiac surgery [8]. Point of care echocardiography is an evolving modality receiving increasing interest in modern anesthesia in recent years. Inconsistent with formal TTE examination, a focused, goal-directed study to answer a particular question is often more appropriate in the perioperative period. Several lines of evidence had demonstrated its positive effect on rescuing patients with circulatory collapse through seeking the etiology of those critical events accurately and in time $[9,10]$. Herein we report two cases of PPCM presenting with left ventricular ejection fraction below 30\% undergoing LSCS in which TTE was used to optimize the fetal and maternal outcome. To our knowledge, this is the first report concerning about anesthetic management for parturients with PPCM undergoing LSCS under the guidance of point of care TTE at bedside.

\section{Case presentation}

\section{Case 1}

A 22 -year-old woman presented as gravida 1 , para 0 , at $38^{+4}$ weeks of gestation with the deterioration of dyspnea on exertion and palpitation over the past several days. The patient was otherwise healthy and developed cough with mucoid sputum at 38th week of pregnancy. She exhibited paroxysmal nocturnal dyspnea, shortness of breath on mild exertion and pedal edema two days later.

On arriving at operation room, the patient was in semi-sitting position on oxygen via nasal tube at $5 \mathrm{~L} / \mathrm{min}$ with pulse oxygen saturation of $93 \%$. She was restlessness and blood pressure $145 / 90$ $\mathrm{mmHg}$, heart rate 140 beats/min with sinus rhythm, and respiratory rate over $25 / \mathrm{min}$. Her hemoglobin was $9.4 \mathrm{~g} / \mathrm{dl}$ with a normal blood profile. According to TTE report from cardiac based echocardiography unit, there was a decline in systolic function manifested by overall global wall motion abnormality. In addition, severe mitral regurgitation, moderate tricuspid regurgitation, and moderate pulmonary hypertension were observed. The left ventricular end diastolic dimension (EDD) and end systolic dimension (ESD) was $5.55,4.80 \mathrm{~cm}$ respectively with the left ventricular ejection fraction (LVEF) $28 \%$. The preoperative echocardiographic screening was performed promptly to differentiate the cause of aggravated dyspnea in the operation room. The bedside echocardiography explicitly showed she had very poor LV contractility with EF less than $25 \%$. Lung ultrasound showed lung sliding sign and bilateral dense B lines over 2 zones which was highly indicative of pulmonary edema. There were no sign of right ventricular strain and massive pleural and pericardium effusion in this patient. She was definitive diagnosed as PPCM with acute onset of congestive heart failure and classified in ASA category IV. She was given high flow oxygen with facemask followed by intravenous injection of $10 \mathrm{mg}$ furosemide. At same time, the invasive blood pressure monitor was established, and arterial blood sample was drawn for blood gas analysis ( $\mathrm{PH} 7.46, \mathrm{PO}_{2} 93 \mathrm{mmHg}, \mathrm{PaCO}_{2} 25 \mathrm{mmHg}$, lactate 1.0mmol/L, HCO3 $17.8 \mathrm{mmol} / \mathrm{L}$ and $\mathrm{BE}-5.0 \mathrm{mmol} / \mathrm{L}$ ). She was judiciously titrated with 0.4 $\mathrm{mg}$ cedilanid and vasodilator (calcium channel blocker, i.e. nicardipine at the infusion rate $3-5 \mathrm{mg} / \mathrm{h}$ ). Afterwards, she was much relieved and echocardiography examination also showed improved LV function with EF approximately $35 \%$. Thereafter, we excluded the intra-cavity thrombus formation in the LV apex which is not uncommon in PPCM with EF less than $35 \%$ and anticoagulation treatment was not mandatory immediately in this case. Therefore, she received the LSCS under spinal anesthesia. The T6 dermal sensory plane blockage was attained $5 \mathrm{~min}$ after intrathecal administration of $10 \mathrm{mg} 0.5 \%$ bupivacaine. A male neonate was delivered with Apgar score 8, 10 at 1, 5 min postbirth respectively. She had good uterus contraction under the titration of oxytocin $10 \mathrm{IU}$ intrauterine injection followed with continuous intravenous instillation at the rate of $3 \mathrm{IU} / \mathrm{h}$. The intraoperative TTE monitor showed persistent improved LV contractility and alleviation in lung alveoli edema. Throughout the surgery, she was hemodynamically stable and calm. The total of $200 \mathrm{~mL}$ of Lactate Ringer's solution and $200 \mathrm{ml}$ normal saline were given intraoperatively. The patient was 100 $\mathrm{mL}$ surplus in fluid balance with $100 \mathrm{~mL}$ urine output and $200 \mathrm{~mL}$ blood loss perioperatively. After surgery, the patient was transferred to ICU for continued treatment and discharged from hospital on the 13 th postnatal day. She was symptom free with a normal ventricular function in 6 months of follow-up.

\section{Case 2}

A 44 -year-old pregnant woman at $27^{+5}$ weeks of pregnancy was admitted to the emergency service with progressing shortness of breath, chest pain and faintness. The patient stated that she had a gradual worsening of shortness of breath over the past two months without seeking any medical help in her hometown. There was no history of pre-existing cardiac illness. When the parturient arrived at operation room, routine monitor was placed including noninvasive blood pressure, pulse oximetry, EKG and temperature. She was hypotensive and tachycardia with restlessness. She was also mild hypoxia with pulse oxygen saturation of $90 \%$ on oxygen at $8 \mathrm{~L} / \mathrm{min}$ via nasal tube. Electrocardiogram showed she had frequent ventricular premature beats and complete right bundle branch block accompanied with left posterior fascicular block. Preoperative transthoracic echocardiogram of the previous day revealed the whole heart cavity was enlarged predominantly in left ventricular (EDD $7.2 \mathrm{~cm}$, ESD $6.05 \mathrm{~cm}$ ). There was a severe decline in systolic function with LVEF of $33 \%$. In addition, severe mitral regurgitation, moderate tricuspid regurgitation, moderate pulmonary hypertension was noted. Oxygen was given by facemask and intravascular catheter was inserted into her left radial artery for invasive blood pressure monitoring and blood gas analysis $\left(\mathrm{PH} 7.52, \mathrm{PO}_{2} 199\right.$ $\mathrm{mmHg}, \mathrm{PaCO}_{2} 27 \mathrm{mmHg}$, lactate $1.0 \mathrm{mmol} / \mathrm{L}$, and $\mathrm{BE}-0.9 \mathrm{mmol} / 1, \mathrm{HCO}_{3}$ $25.1 \mathrm{mmol} / \mathrm{L}$ ). The bedside point of care TTE was ordered immediately.

identified with cardiogenic shock with severely suppression of LV contractility (EF 27.4\%). The large pericardium effusion leading to tamponade and pulmonary embolism were excluded with Apical four and subxiphoid views. She received inotropic infusion (Dopamine with Dobutamine at dose of $10 \mathrm{microgram} / \mathrm{kg} / \mathrm{min}$ ) without rapid alleviation in symptom of acute heart failure after $30 \mathrm{~min}$ circulatory support. Cesarean section under general anesthesia was planned. Considering the suppressed LV contractility, we put the patient into sleep with low dose of etomidate $10 \mathrm{mg}$ and remifentanil 40 microgram followed with vecuronium $8 \mathrm{mg}$ facilitating orally tracheal intubation. The Sellick maneuver was applied before the oro-tracheal tube was in position and the tracheal tube cuff was inflated to seal the airway. The patient's lungs were ventilated with following parameters: tidal volume of 500 $\mathrm{ml}$, respiration rate 12 times/ $\mathrm{min}$, and positive-end expiratory pressure (PEEP) $5 \mathrm{cmH}_{2} \mathrm{O}$. Intraoperative TTE may also be useful for evaluating dynamic changes in cardiac performance and response to inotropes and fluid loading. In fear of perioperative significant volume alteration, we assessed the IVC dimension over the respiratory circle from right upper quadrant of abdomen at anterior axillary line. The dimension of IVC was barely changed after baby and placenta delivery in this patient. A female preterm neonate weighted $1.05 \mathrm{~kg}$ was delivered with Apgar score of 3 at 1, 5 min post-birth. Standardized neonate cardiac pulmonary resuscitation procedure was immediately implemented for 60 min with no improvement of pulse oxygen saturation less than $60 \%$ 
and heart rate below 60 beat/min. Her family made final decision on abandoning further medical intervention. The parturient had good uterus contraction under the titration of oxytocin and was transferred to the ICU after LSCS. The total of $300 \mathrm{~mL}$ of Lactate Ringer's solution was given to this parturient, whereas urine output and total blood loss was $700 \mathrm{~mL}$ and $400 \mathrm{ml}$ respective intraoperatively. She was apparently $800 \mathrm{~mL}$ deficit in fluid balance during LSCS. The patient was treated with furosemide, vasodilator, beta-blockers and antibiotic therapy in ICU and department of Cardiology for controlling the heart failure and infection prevention. The symptoms of cardiac dysfunction were significantly improved, and she was discharged from hospital on the 20th postnatal day. The classical features of PPCM were alleviated in 6 months follow-up after discharge from hospital.

\section{Discussion}

Peripartum cardiomyopathy (PPCM) is a rare but potentially devastating disease of unknown origin [11]. Those patients had no other obvious causes of heart failure and no pre-existing heart disease. Several mechanisms have been proposed to be involved in the development of PPCM including the theory of selenium deficiency [11], autoimmunity [12], viral infection [13] or genetic factors [14]. Although the incidence is low patients with an $\mathrm{EF}$ about $25 \%$ had a mortality rate of $50 \%$ in two years [15]. Albeit early diagnosis is important for successful treatment of PPCM, it is commonly difficult to access because the initial symptoms of PPCM, such as dyspnea, fatigue, and peripheral edema are similar to that of late stage of pregnancy [12-14]. Obstetric management for those parturients includes control of symptom of chronic heart failure and the choice of the timing of pregnancy cessation.

Anesthesiologist-operational perioperative echocardiography plays an important role in excluding the comorbidities associated with pregnancy-related circulatory compromise notwithstanding the diagnosis of PPCM still requires thorough formal echocardiographic examination of heart structure by experienced practitioners to exclude pre-existing cardiac anomaly. First of all, the pre-anesthesia echocardiographic examination was helpful in diagnosis for undifferentiated hemodynamic instability and assessment of the patient's status. We can promptly exclude the other critical events resulting in cardiovascular collapse including amniotic fluid embolism, tamponade, and massive pleural effusion and thrombus formation in the left ventricle, which are not uncommon during labor. Although left heart failure was highly suspected in those two patients, we couldn't tell whether the heart failure was progressively deteriorated with the time elapse or other comorbidities existed without the superficial thoracic ultrasound examination in the operation room. Moreover, the point of care echocardiography was a useful tool to identify the global wall motion abnormality with qualitative (visual assessment or M-model modality) or quantitative analysis of stroke volume respectively. The variables of $\mathrm{E}$ point of mitral valve anterior leaflet septal separation (EPSS) and ejection fraction (EF) of left ventricle using M modality were commonly used to evaluate the contractility of left ventricle. Inconsistent with the quantitative calculation of stroke volume through calculating the product of velocity time interval and diameter of left ventricle outflow tract, the measurement of EPSS or EF with teichholz's formula was relatively less time-consuming and facilitated in rapid cardiac function assessment. These two parturients suffered severely myocardial suppression with EF below $30 \%$ on arrival at operation room identified with TTE, therefore inotropic support was initiated without delay (Figures 1 and 2).

Issues such as whether the pregnancy should be allowed to reach term and the mode of delivery need to be addressed by the team
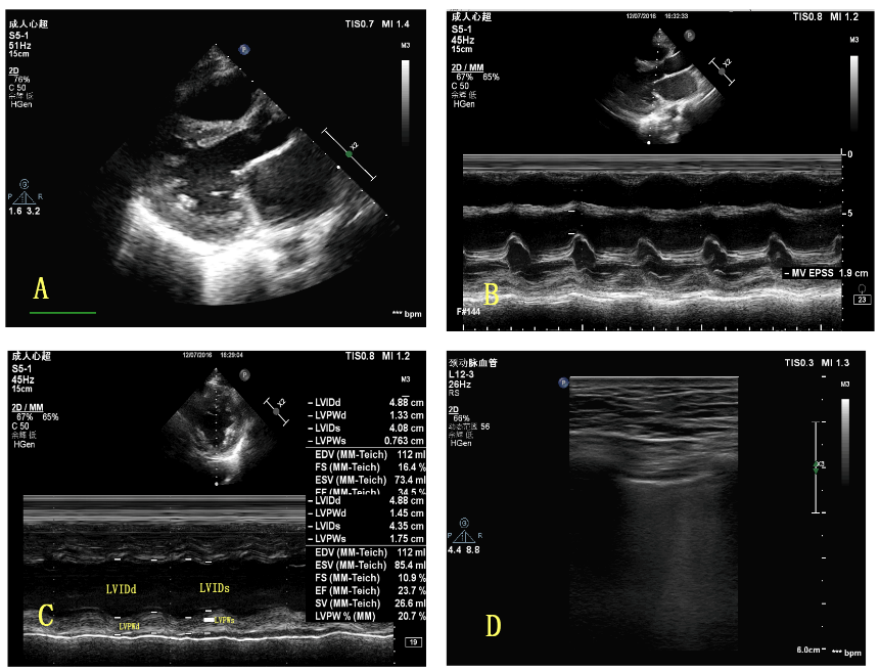

Figure 1. Dilated left ventricle and left atrium were shown from parasternal long axis view in A; EPSS was measured with M-modality from parasternal long axis view in B; Left ventricle $\mathrm{EF}$ was measured with M-modality from parasternal short axis view at papillary muscle level in C; Dense B Lines were shown over 2 zones of bilateral Lungs in D
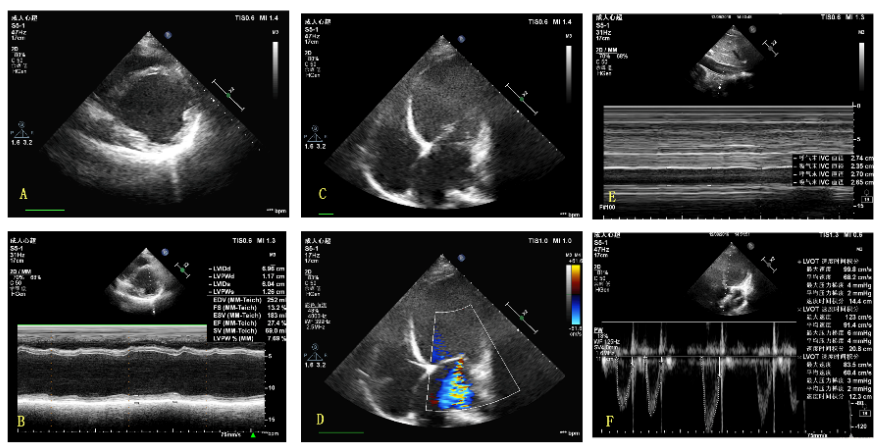

Figure 2. Dilated Left ventricle and severely reduced EF were shown from parasterna short axis view at papillary muscle level with 2-D (A) and M modality (B) respectively; the enlarged Left ventricle and severe mitral regurgitation were shown from apex 4 chambers view with 2- D (C) and color Doppler modality (D). The in-collapsibility of IVC over respiratory circles was shown from subxiphoid view (E); the quantitative assessment of the VTI of LVOT from Apical 5 chambers view with pulse wave Doppler (F)

comprising of obstetric, cardiology, pediatric, and anesthesiology according to the patient's medical status on a periodical basis. Anesthesiologists are commonly involved during the delivery phase of those parturients with PPCM by providing anesthesia for cesarean section. Either of general or regional anesthesia have been successfully applied in PPCM in multiple of case reports $[16,17]$. There is no evidence supporting that general is superior to the neuraxial anesthesia, or vice versa. Pre-operative TTE was considered as the essential factor responsible for selection of anesthetic modality for patients in view of evaluating the inotropic responsiveness to therapeutic intervention. In case-1, the symptoms of heart failure were subsidy and the contractility of left ventricle improved confirmed by echocardiography monitoring following inotropic support and vasodilator treatment. Concerning about the alleviation in acute congestive heart failure and normal coagulation profile in this parturient, spinal anesthesia was administrated with judicious titration of $10 \mathrm{mg}$ bupivacaine. On the contrary, the hemodynamic parameters didn't show any significant improvement under the aggressive inotropic intervention in case-2, we decided to conduct general anesthesia with mechanic ventilation for further hemodynamic optimization through providing better oxygenation and intensified interventions. Moreover, TTE can be 
Su MY (2018) Application of point of care transthoracic echocardiography in parturient with peripartum cardiomyopathy undergoing lower segmental cesarean section- 2 cases report

utilized to screen the LV thrombus formation in PPCM with severely reduced EF. Once the detection of thrombus existing in LV cavity anticoagulation with heparin will commence without delay for fear of systemic thromboembolism. It, therefore, is not suitable to choose neuraxial block for those patients receiving anticoagulation intervention unless the surgery had to be postponed 12-24 h after an initial dose of heparin. The choice of anesthesia for those parturients should be individualized according to the high-quality TTE image to interpreting the cardiac functioning and hemodynamic status of parturients.

In addition, the perioperative use of TTE during surgery is an ideal noninvasive continuous monitor of RV filling through inferior vena cava (IVC) dimension measurement with a modified maneuver at anterior axilla line. The IVC respiratory variation and its dimension were applied to closely assess the volume status especially after placenta delivery in fear of auto transfusion leading to volume overload and deterioration of heart function. There is no prominent IVC dilation and reduced respiratory variation after placenta delivery and increased tricuspid regurgitation in case-2, therefore we didn't give any diuretics or nitroglycerin to reduce intravenous return to right atrium. However the auto-transfusion concept was currently receiving challenges from the study by Kuhn JC [18]. They did observe the decreased cardiac output by $32 \%$, stroke volume by $44 \%$, increased heart rate by $52 \%$ compared to baseline in healthy parturients after virginal delivery as opposing to volume expansion due to auto-transfusion proposed by previous studies. Therefore, the volume statue of parturient (especially undergoing cesarean section) should be determined by the balance between the blood loss and auto infusion or the use of oxytocin during the phase of placenta delivery, which can be assessed with echocardiography with ease.

Lastly, great caution should be taken to oxytocin use in these critical ill patients. The deleterious effect of coronary arterioles spam induced by oxytocin may be catastrophic in patients with PPCM. We use loading dose of $10 \mathrm{IU}$ intramyometrial injection and followed with iv infusion rate at $3 \mathrm{IU} / \mathrm{h}$. The use of TTE in these patients helps in describing the biventricular function, systolic and diastolic status of the heart, any ischemia developing in the form of new regional wall motion abnormality during surgery. Under this regime and route of oxytocin administration, we didn't observe any the regional wall motion abnormality and hemodynamic fluctuation in these two parturients and acquired the satisfactory uterus contraction meantime.

\section{Conclusion}

The parturient with PPCM undergoing cesarean section is a big challenge for anesthesiologists. The hemodynamic assessment and perioperative anesthetic management require much knowledge in pathophysiology of PPCM. The point of care echocardiography is helpful in assistance in physical examination, avoid unnecessary delays or cancellation, obtain rapid diagnostic information in the operating room and assess the volume status as well as response to therapy for patients with PPCM.

\section{Acknowledgement}

$$
\text { Not applicable }
$$

\section{Funding}

This study was supported by the Project of Six Talent Summit of Jiangsu Province (2016-WSN-147), the Project of Bureau of Science and Technology of Nanjing (201503019), Medical Science and Technique Development Foundation of Nanjing Municipal Government (No.
QRX17013), and the Project of Key Medical Discipline of Jiangsu Province for 13rd 5-Year Plan Development.

\section{Competing interest}

The authors declare that they have no competing interests.

\section{References}

1. Bauersachs J, Arrigo M, Hilfiker-Kleiner D, Veltmann C, Coats AJ, et al. (2016) Current management of patients with severe acute peripartum cardiomyopathy: practical guidance from the Heart Failure Association of the European Society of Cardiology Study Group on peripartum cardiomyopathy. Eur J Heart Fail 18: 10961105. [Crossref]

2. Lim CP, Sim DK (2013) Peripartum cardiomyopathy: experience in an Asian tertiary centre. Singapore Med J 54: 24-27. [Crossref]

3. Johnson-Coyle L, Jensen L, Sobey (2012) Peripartum cardiomyopathy: review and practice guidelines. Am J Crit Care 21: 89-99. [Crossref]

4. Sliwa K, Hilfiker-Kleiner D, Petrie MC, Mebazaa A, Pieske B, et al. (2010) Current state of knowledge on aetiology, diagnosis, management, and therapy of peripartum cardiomyopathy: a position statement from the Heart Failure Association of the European Society of Cardiology Working Group on peripartum cardiomyopathy. Eur J Heart Fail 12: 767-778. [Crossref]

5. Ramaraj R, Sorrell VL (2009) Peripartum cardiomyopathy: Causes, diagnosis, and treatment. Cleve Clin J Med 76: 289-296. [Crossref]

6. Prasad GS, Bhupali A, Prasad S, Patil AN, Deka Y (2014) Peripartum cardiomyopathy - case series. Indian Heart J 66: 223-226. [Crossref]

7. Hilfiker-Kleiner D, Haghikia A, Nonhoff J, et al. (2015) Peripartum cardiomyopathy: current management and future perspectives. Eur Heart J 36: 1090-1097. [Crossref]

8. Canty DJ, Royse CF (2009) Audit of anaesthetist-performed echocardiography on perioperative management decisions for non-cardiac surgery. Br J Anaesth 103: 352358. [Crossref]

9. Tomaszuk-Kazberuk A, Lisowska A, Sobkowicz B, Mlodawska E, Lopatowska P, et al. (2012) Is establishing diagnosis of perivalvular abscess still difficult? Kardiol Pol 70: $1164-1166$.

10. Glaser JJ, Cardarelli C, Galvagno S Jr, Scalea TM, Murthi SB (2016) Bridging the gap Hybrid cardiac echo in the critically ill. J Trauma Acute Care Surg 81: S157-157S161. [Crossref]

11. Pearson GD, Veille JC, Rahimtoola S, Hsia J, Oakley CM, et al. (2000) Peripartum cardiomyopathy: National Heart, Lung, and Blood Institute and Office of Rare Diseases (National Institutes of Health) workshop recommendations and review. JAMA 283: 1183-1188. [Crossref]

12. Abboud J, Murad Y, Chen-Scarabelli C, Saravolatz L, Scarabelli TM (2007) Peripartum cardiomyopathy: a comprehensive review. Int J Cardiol 118: 295-303. [Crossref]

13. Elkayam U (2011) Clinical characteristics of peripartum cardiomyopathy in the United States: diagnosis, prognosis, and management. J Am Coll Cardiol 58:659-670. [Crossref]

14. Smith IJ, Gillham MJ (2009) Fulminant peripartum cardiomyopathy rescue with extracorporeal membranous oxygenation. Int J Obstet Anesth 18: 186-188. [Crossref]

15. Cuenza LR, Manapat N, Jalique JR (2016) Clinical Profile and Predictors of Outcomes of Patients with Peripartum Cardiomyopathy: The Philippine Heart Center Experience. ASEAN Heart J 24: 9. [Crossref]

16. Kim HY, Jeon HJ, Yun JH, Lee JH, Lee GG, et al. (2014) Anesthetic experience using extracorporeal membrane oxygenation for cesarean section in the patient with peripartum cardiomyopathy: a case report. Korean J Anesthesiol 66: 392-397. [Crossref]

17. Kapoor PM, Goyal S, Irpachi K, Smita B (2014) Importance of transesophageal echocardiography in peripartum cardiomyopathy undergoing lower section cesarean section under regional anesthesia. J Anaesthesiol Clin Pharmacol 30: 427-429. [Crossref]

18. Kuhn JC, Falk RS, Langesæter E (2017) Haemodynamic changes during labour: continuous minimally invasive monitoring in 20 healthy parturients. Intern $\mathrm{J}$ Obstet Anesthesia 31: 74-83.

Copyright: (C2018 Su MY. This is an open-access article distributed under the terms of the Creative Commons Attribution License, which permits unrestricted use, distribution, and reproduction in any medium, provided the original author and source are credited. 